\title{
К ТЕОРИИ НЕЛИНЕЙНЫХ ВОЛН ДЕФОРМАЦИИ В НЕОДНОРОДНОЙ СРЕДЕ
}

\author{
(Представлена Н. Алумяэ)
}

Особенностью нелинейных волн деформации является их способность при распространении испытывать разрыв в случае гладких краевых условий. Если же нелинейность сопровождается и другими физическими эффектами, искажающими профиль волны, то анализ нелинейного процесса необходимо проводить с учетом дополнительных условий. Покажем, что такая ситуация имеет место при распространении нелинейных волн деформации в неоднородной среде.

Одномерный волновой процесс в неоднородной среде с учетом физической и геометрической нелинейностей описывается матричным уравнением

$$
I \frac{\partial \vec{U}}{\partial t}+A(X, \vec{U}) \frac{\partial \vec{U}}{\partial X}+K(X) \frac{\partial \vec{F}}{\partial X}=0
$$

где $\vec{U}$ - неизвестный вектор полевых величин, $X, t$ - лагранжевы переменные, $A, K-$ известные матрицы-коэффициенты, $\vec{F}-$ известный вектор, $I-$ единичная матрица. Требуется найти решение уравнения (1) при условиях

$$
\vec{U}(X, 0)=0, \vec{U}(0, t)=\vec{\psi}(t), \vec{\psi}(t)=\vec{\psi}_{1}(t)\left[H(t)-H\left(t-t_{0}\right)\right],
$$

где $H(t)$ - функция Хевисайда, $t_{0}$ - продолжительность импульса.

Чтобы найти асимптотические решения уравнения (1) при условиях (2), построим с помощью лучевого метода для нелинейных волн $\left[{ }^{1}\right]$ уравнение переноса для каждой определенной волны, имея в виду все члены разложения

$$
\vec{U}=\vec{U}_{0}+\varepsilon \vec{U}_{1}+\ldots
$$

где $\varepsilon-$ малый параметр. Это сразу приведет к разложению $A=$ $=A_{0}(X)+\varepsilon A_{1}(X, \vec{U})+\ldots$ Тогда для продольной волны получим уравнение переноса 1-го порядка относительно амплитудного фактора $\alpha$

$$
\alpha_{\tau}+a_{1}(\tau) \alpha \alpha_{\xi}+a_{2}(\tau) \alpha=0,
$$

для которого решим задачу Коши с начальным условием 


$$
\alpha(0, \xi)=\varphi(\xi)=\vec{l} \psi(\xi),
$$

при этом амплитудный фактор $\alpha$ связан с вектором $\vec{U}_{0}$ соотношением

$$
\vec{U}_{0}=\alpha \vec{r} .
$$

Здесь $\xi=t-\int^{X} c^{-1} d x, \tau=\varepsilon X-$ лучевые координаты, $c=c(X)-$ скорость продольной волны, индексы $\xi, \tau$ означают дифференцирование, $a_{1}(\tau), a_{2}(\tau)-$ коэффициенты, $l, \vec{r}-$ левый и правый собственные векторы матрицы $A_{0}$ для собственного значения $c$. Условия гиперболичности уравнения (1) гарантируют и гиперболичность уравнения (4), решение которого сводится к решению системы двух обыкновенных дифференциальных уравнений $\left[{ }^{2}\right]$

$$
\frac{d \xi}{d \tau}=a_{1}(\tau) \alpha, \quad \frac{d \alpha}{d \tau}=-a_{2}(\tau) \alpha .
$$

Учитывая условие (5), получим

$$
\begin{aligned}
& \alpha=\exp \left(-\int_{0}^{\tau} a_{2}(y) d y\right) \varphi\left(\xi_{0}\right), \\
& \xi=\xi_{0}+\varphi(\xi) \int_{0}^{\tau} a_{1}(y) \exp \left(-\int_{0}^{\tau} a_{2}(y) d y\right) d y, \\
& \xi=\xi_{0} \quad \text { при } \tau=0 .
\end{aligned}
$$

Соотношения (6) описывают семейство характеристических кривых, имеющих в случае возникновения и распространения разрыва огибающую, определяющую его фронт. Эта огибающая удовлетворяет полученному дифференцированием второго из соотношений (6) по параметру छо выражению

$$
\varphi_{\xi_{0}}\left(\xi_{0}\right)=-\left[\int_{0}^{\tau} a_{1}(y) \exp \left(-\int_{0}^{\tau} a_{2}(y) d y\right) d y\right]^{-1},
$$

которое позволяет оценить поведение волны в нелинейной среде. Обозначив правую часть равенства (7) через $\varphi_{\xi}^{*}$, сформулируем на основе изложенного выше теорему, справедливую с точностью уравнения переноса 1-го порядка (4).

Т е о р е м а. Пусть действительный градиент $\varphi_{\xi_{0}}\left(\xi_{0}\right)$ удовлетворяет одному из условий
a) $\varphi_{\xi_{0}}\left(\xi_{0}\right)<\varphi_{\xi}^{*}(\xi)$;
б) $\varphi_{\xi_{0}}\left(\xi_{0}\right)=\varphi \dot{\xi}(\xi)$;
в) $\varphi \xi_{0}\left(\xi_{0}\right)>\varphi_{\xi}^{*}(\xi)$.

Тогда при распространении продольной волны в неоднородной среде возможны следующие случаи: 1) разрыв не возникает или амплитуда существующего разрыва уменьшается, 2) ее профиль остается постоянным, 3) разрыв возникает или амплитуда существующего разрыва увеличивается.

Исходя из теоремы, назовем $\varphi_{\xi}^{*}(\xi)$ критическим градиентом - понятие, введенное в [ $\left.{ }^{3}\right]$ для случая $a_{1}=1, a_{2}=$ const $>0$.

Перейдем теперь к конкретизации модели (1). Предположим, что

$$
\lambda+2 \mu=\left(\lambda_{0}+2 \mu_{0}\right) f(X), \quad c^{2}=c_{0}^{2} g(X), \quad f(X)>0, \quad g(X)>0,
$$


где $\lambda, \mu-$ параметры Ляме, $c^{2}=(\lambda+2 \mu) \varrho^{-1}-$ скорость продольной волны, 巳 - плотность недеформированной среды, индекс определяет значения указанных величин при $X=0$. Тогда имеем

$$
\begin{aligned}
& a_{1}=\frac{3}{2}\left(f(X)+m_{0}\right)\left(\varepsilon c^{2} f(X)\right)^{-1}, \\
& a_{2}=\frac{1}{2}(\varepsilon f(X))^{-1} \partial f(X) / \partial X, \\
& m_{0}=2\left(v_{1}+v_{2}+v_{3}\right)\left(\lambda_{0}+2 \mu_{0}\right)^{-1},
\end{aligned}
$$

где $v_{i}, i=1,2,3$ - модули упругости третьего порядка. Подчеркнем, что появление критического градиента, как вытекает из этих соотношений, связано не с изменением скорости, а с изменением модулей упругости, которое приводит к таким же изменениям амплитуды волны, как и в случае волновых процессов с цилиндрической и сферической симметрией. Поскольку эти соотношения довольно громоздки, ограничимся случаем слабой неоднородности

$$
f(X)=1+\varepsilon f_{1}(X), \quad g(X)=1+\varepsilon g_{1}(X) .
$$

Получим

$$
A=A_{0}+\varepsilon A_{11}(X)+\varepsilon A_{12}(\vec{U})+, \ldots, A_{0}=\text { const, }
$$

и уравнение переноса 1-го порядка примет вид

$$
\alpha_{\tau}+a_{1} \alpha \alpha_{\xi}+a_{3} \alpha_{\xi}+a_{2} \alpha=0 .
$$

Используя безразмерную запись, имеем

$$
\begin{gathered}
\tau=\frac{1}{2}|k| \alpha_{c} X c_{0}^{-1} \tau_{c}^{-1}, \quad \xi=\left(c_{0} t-X\right) \tau_{c}^{-1}, \\
a_{1}=\operatorname{sign} k, a_{2}=\tau_{c} c_{0}|k|^{-1} \alpha_{0}^{-1} \varepsilon \partial f_{1}(X) / \partial X, \\
a_{3}=c_{0}|k|^{-1} \alpha_{0}^{-1} \varepsilon g_{1}(X), \quad k=3\left(1+m_{0}\right),
\end{gathered}
$$

где $\alpha_{0}$ - максимальная амплитуда, $\tau_{c}-$ характерная длина импульса. Следуя $\left[{ }^{4}\right]$, введем в рассмотрение новую переменную

$$
\zeta=\xi+\int_{0}^{t} a_{3}(y) d y .
$$

Тогда уравнение (8) примет вид

$$
\alpha_{\tau}+a_{1} \alpha \alpha_{\zeta}+a_{2} \alpha=0
$$

с начальным условием

$$
\alpha(0, \zeta)=\varphi(\zeta)
$$

Если $f_{1}(X)$ является линейной функцией с коэффициентом пропорциональности $k_{0}$, возможно дальнейшее упрощение формул (7) и (9). Имея в виду, что в твердых средах обычно $\operatorname{sign}\left(1+m_{0}\right)=-1$, из соотношений (7) и (9) получим значение критического градиента

$$
\varphi_{\zeta}^{*}=a_{2}=\tau_{c} c_{0}|k|^{-1} \alpha_{0}^{-1} \varepsilon k_{0} .
$$


Для этого случая выражения (6) и (7) существенно упрощаются, что позволяет дополнить теорему двумя следствиями.

Сл едствие 1. В среде, в которой модуль упругости второго порядка увеличивается $\left(\varepsilon k_{0}>0\right)$ в направлении распространения волны, можно ожидать образования разрыва в результате увеличения сжимающего напряжения или уменьшения растягивающего напряжения при выполнении условия

$$
\varphi_{\zeta}(\zeta)>\dot{\varphi}_{\zeta}
$$

С лед ств и е 2. В среде, в которой модуль упругости второго порядка уменьшается $\left(\varepsilon k_{0}<0\right)$ в направлении распространения волны, можно ожидать образования разрыва в результате увеличения сжимающцего напряжения или уменьщения растягизающего напряжения при выполнении условий

a) $\varphi_{\zeta}(\zeta)>\left|\varphi_{\zeta}^{*}\right|, \quad 0<\tau<0,6931\left|\varphi_{\zeta}^{*}\right|^{-1}$;

б) $\varphi_{\zeta}(\zeta)<\left|\varphi_{\zeta}^{*}\right|, \quad \tau>0,6931\left|\varphi_{\zeta}^{*}\right|^{-1}$.

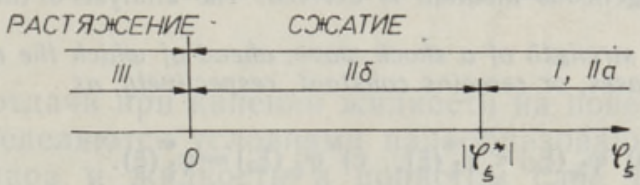

Последние условия обусловлены множителем [exp $\left.\left(\left|a_{2}\right| \tau\right)-1\right]$ в соотношении огибаюшей характеристик (7). Области, на которые распространяются эти следствия, показаны на рисунке. Область I по оси $\varphi_{\xi}$ определяется следствием 1 , области ІІа и ІІб - следствием 2, а область III $\left(\varphi_{\zeta}(\zeta)<0\right)$ не содержит разрывных решений. Напомним, что эти результаты справедливы для случая $1+m_{0}<0$.

K уравнению переноса (10) приводятся и некоторые задачи распространения волн в релаксирующей среде [ $\left.{ }^{5}\right]$ и в конечном интервале с учетом радиации энергии от его концов $\left[{ }^{6}\right]$.

\section{Л ИТЕР А Т Р Р А}

1. Эн гель брех т Ю. К. О теории нелинейных волновых процессов в диссипативной среде. - Изв. АН СССР, МТТ, 1977, вып. 2, с. 142-148.

2. Ро ждественский Б. Л., Яненко Н. Н. Системы квазилинейных уравнений и их приложения к газовой динамике, М., 1968.

3. У и з е м Дж. Линейные и нелинейные волны. М., 1977.

4. A s a n o, N. Wave propagation in non-uniform media. - Suppl. Progr. Theor. Phys., 1974 , N 55, p. 52-79.

5. Руденко О. В., Солуян С. И. Теоретические основы нелинейной акустики. М., 1975.

6. Mortell, M. P. The evolution of nonlinear standing waves in bounded media. ZAMP, 1977 , v. $28, \mathrm{~N} 1$, p. $33-46$.

Ннститут кибернетики Академии наук Эстонской ССР
Поступила в редакцию $4 / \mathrm{I} 1978$ 


\section{J. ENGELBRECHT}

\section{MITTEHOMOGEENSES KESKKONNAS LEVIVATE MITTELINEAARSETE DEFORMATSIOONILAINETE TEOORIAST}

On tuletatud ühemõōtmelise pikilaine transpordivõrrand, mis võimaldab modelleerida mittelineaarset lainelevi mittehomogeenses keskkonnas. Transpordivõrrandi analüüsi põhjal on formuleeritud teoreem, mis kriitilise gradiendi mõiste abil määrab pikilaine katkevuse arengu iseloomu. Esitatud transpordivõrrandi koefitsiendid kehtivad konkreetse nõrga mittehomogeensuse korral. Võrrandi analüüsist järeldub, et katkevuse tekkimine mittehomogeenses keskkonnas sõltub laine leviku suunast. On määratud kriitilise gradiendi väärtused.

\section{J. ENGELBRECHT}

\section{ON THE THEORY OF NONLINEAR DEFORMATION WAVES IN A NONHOMOGENEOUS MEDIUM}

The transport equation of the 1st order for a longitudinal deformation wave in an onedimensional nonhomogeneous medium is derived. The analysis of this equation permits to formulate a

Th e or em: The strength of a shock wave, ahead of which the material is in uniform state, increases, decreases, or remains constant, respectively, as
a) $\varphi_{\xi_{0}}\left(\xi_{0}\right)>\varphi_{\xi}^{*}(\xi)$,
b) $\varphi_{\xi_{0}}\left(\xi_{0}\right)<\varphi_{\xi}^{*}(\xi)$,
c) $\varphi_{\xi_{0}}\left(\xi_{0}\right)=\varphi_{\xi}^{*}(\xi)$.

The coefficients of the transport equation are obtained for the case of weak nonhomogeneity. In this case it is shown that the shock wave development conditions given above depend on the shock wave propagation direction.

Here $\varphi_{\xi}^{*}(\xi)$ is the critical deformation gradient, $\varphi_{\xi_{0}}\left(\xi_{0}\right)-$ the deformation gradient at the considered moment. 